\title{
Alcohol consumption, drinking patterns, and cognitive function in older Eastern European adults OPEN
}

Pia Horvat, $\mathrm{PhD}$

Marcus Richards, PhD

Ruzena Kubinova, MD

Andrzej Pajak, MD, PhD

Sofia Malyutina, MD,

$\mathrm{PhD}$

Sergey Shishkin, MD

Hynek Pikhart, PhD

Anne Peasey, PhD

M.G. Marmot, FRCP

Archana Singh-Manoux,

PhD

Martin Bobak, MD, PhD

Correspondence to

Dr. Horvat:

pia.horvat.10@ucl.ac.uk

Supplemental data at Neurology.org

\section{ABSTRACT}

Objective: To investigate associations of frequency, quantity, binge, and problem drinking with cognitive function in older Eastern European adults.

Methods: The investigation included 14,575 participants, aged 47 to 78 years at cognitive assessment in 2006-2008 from Novosibirsk (Russia), Krakow (Poland), and 6 Czech towns participating in the HAPIEE (Health, Alcohol, and Psychosocial Factors in Eastern Europe) prospective cohort study. Average response rates were 59\% at baseline (2002-2005) and 63\% in 2006-2008. Alcohol consumption was assessed at baseline and in 2006-2008. Cognitive tests included immediate and delayed word recall, semantic fluency (animal naming), and letter cancellation. Associations between alcohol indices and cognitive scores were analyzed crosssectionally (all measures from 2006 to 2008) and prospectively (alcohol and covariates from 2002 to 2005 and cognition from 2006 to 2008).

Results: In cross-sectional analyses, nondrinkers had lower cognitive scores and female moderate drinkers had better cognitive performance than light drinkers. Heavy, binge, and problem drinking were not consistently associated with cognitive function. Few associations were replicated in prospective analyses. Participants who stopped drinking during follow-up had worse cognition than stable drinkers; in men, regression coefficients (95\% confidence interval) ranged from $-0.26(-0.36,-0.16)$ for immediate recall to $-0.14(-0.24,-0.04)$ for fluency.

Conclusion: Regular and episodic heavy drinking were not consistently associated with cognitive function. Worse cognition in participants who stopped drinking during follow-up suggests that inclusion of less healthy ex-drinkers may partly explain poorer cognition in nondrinkers. Neurology ${ }^{\circledR}$ 2015;84:287-295

\section{GLOSSARY}

CAGE = cutting down, annoyance by criticism, guilty feeling, and eye-openers; CVD = cardiovascular disease; GFQ = graduated frequency questionnaire; HAPIEE = Health, Alcohol, and Psychosocial Factors in Eastern Europe.

Cognitive function is essential for well-being and independent living and is a strong predictor of dementia. ${ }^{1}$ Better cognitive test performance as well as lower risk of all types of dementia in light to moderate drinkers than abstainers has been widely reported by observational studies, ${ }^{2}$ but more research is needed to better understand whether the observed relationship is causal or merely associational.

Chronic heavy alcohol misuse is known to cause neurocognitive impairment, but results of population-based studies in older adults are inconsistent, with some showing an inverted U-shaped association (i.e., worse cognition in heavy drinkers and abstainers), ${ }^{3,4}$ some showing a linear protective effect, ${ }^{5}$ and some showing subgroup-specific ${ }^{6}$ or cognitive domain-specific associations, ${ }^{3,7}$ although a recent study reported faster cognitive decline in heavy vs moderate

\footnotetext{
From the Department of Epidemiology \& Public Health (P.H., H.P., A. Peasey, M.G.M., A.S.-M., M.B.), and MRC Unit for Lifelong Health and Ageing at UCL (M.R.), University College London, UK; National Institute of Public Health (R.K.), Prague, Czech Republic; Department of Epidemiology and Population Studies (A. Pajak), Jagellonian University Collegium Medicum, Krakow, Poland; Institute of Internal and Preventive Medicine Siberian Branch under the Russian Academy of Medical Sciences (S.M., S.S.), Novosibirsk; Novosibirsk State Medical University (S.M.), Novosibirsk, Russia; and INSERM (A.S.-M.), U1018, Hopital Paul Brousse, Villejuif, France.

Go to Neurology.org for full disclosures. Funding information and disclosures deemed relevant by the authors, if any, are provided at the end of the article. The Article Processing Charge was paid by Wellcome Trust.

This is an open access article distributed under the Creative Commons Attribution License, which permits unrestricted use, distribution, and reproduction in any medium, provided the original work is properly cited.
} 
drinkers. ${ }^{8}$ These inconsistencies may partly reflect the relatively small numbers of heavy drinkers in most studies. ${ }^{9}$

Potential effects of regular or episodic heavy drinking (binge drinking) on cognition have received less empirical attention. We identified 2 reports from one cohort, in which binge drinking was found to increase the risk of cognitive impairment ${ }^{10}$ and dementia. ${ }^{11}$ There is emerging evidence that binge drinking independently increases cardiovascular disease (CVD) risk, ${ }^{12}$ and because CVD is associated with cognitive aging, ${ }^{13}$ a link between binge drinking and cognitive function seems plausible.

Our aim was to investigate the associations of quantity, frequency, binge, and problem drinking with cognitive function in older adults, using data from 3 Eastern European populations, where alcohol is an important cause of morbidity and premature mortality. ${ }^{14}$

METHODS Study populations and participants. The HAPIEE (Health, Alcohol, and Psychosocial Factors in Eastern Europe) Study is a multicenter prospective cohort study conducted in Novosibirsk (Russia), Krakow (Poland), and 6 Czech towns. Details of the study protocol have been reported elsewhere. ${ }^{15}$ Briefly, 28,947 men and women aged 45 to 69 years at baseline (2002-2005) were randomly selected from population registers and electoral lists (Novosibirsk). Response rates were $61 \%$ in Krakow and Novosibirsk and 55\% in Czech towns. At baseline, participants completed an extensive health questionnaire and a brief physical examination. Russian and Czech participants were invited for examination in a clinic, while Polish participants were visited and examined in their homes. Reexamination of participants was completed in 2006-2008 (response rate 63\%). At baseline, cognitive tests were performed on all participants older than 60 years, and in a random sample of approximately $20 \%$ of adults aged 45 to 59 years $(n=12,594)$. At reexamination, cognition was assessed in all returning participants $(\mathrm{n}=$ 17,543). The eligible sample for this study consisted of all participants with cognitive data at reexamination; for $54 \%$ ( $\mathrm{n}=$ 9,436 ) of them, it was their first assessment of cognitive function.

Standard protocol approvals, registrations, and patient consents. The study was approved by ethics committees at University College London and University College Hospital and local ethics committees in each participating center. All participants gave written informed consent.

Measurements. Cognitive function (assessed 2006-2008). Cognitive function was assessed by trained nurses using 4 neuropsychological tests. First, 10-word list recall was used to assess verbal memory and learning. Word lists, consisting of $10 \mathrm{com}$ mon nouns, were the same in each center. Immediate recall was assessed by correctly recalled words summed over 3 consecutive 1-minute trials (range 0-30). Delayed recall was assessed after an interval during which other cognitive tests were administered (range 0-10). Second, verbal fluency was assessed by asking participants to name as many animals as possible within $1 \mathrm{~min}$ ute. Third, letter cancellation test was used to assess attention, mental speed, and concentration. Participants were instructed to cross out 2 target letters, P and W (P and $W$ in Novosibirsk), embedded in a random letter grid as quickly and as accurately as possible within 1 minute (range 0-65).

Alcohol consumption (assessed 2002-2005 and 20062008). Self-reported alcohol intake in the past year was measured using the graduated frequency questionnaire (GFQ). ${ }^{16}$ The baseline questionnaire contained 9 mutually exclusive categories of frequency (ranging from "never" to "almost every day") and 6 mutually exclusive categories of amounts, expressed in local units of beer, wine, and spirits $(\geq 10,7-9,5-6,3-4,1-2$, and 0.5 drink; 1 drink equals $0.5 \mathrm{~L}$ of beer, $0.2 \mathrm{~L}$ of wine, and $0.05 \mathrm{~L}$ of spirits or $20 \mathrm{~g}$ of ethanol). Because only 3 categories of amounts $(\geq 5,3-4,2-0.5$ drinks) were used at reexamination, the data were harmonized across the waves. Several alcohol indices were derived from harmonized GFQ data for both data waves. First, drinking frequency in the past year was categorized as follows: never, $<1$ monthly, 1-3 times monthly, 1-4 times weekly, and $5+$ times weekly. Second, the frequency of consuming a given number of alcoholic drinks in the past year was converted into total amount of alcohol consumed in grams and divided by 365 to obtain an estimate of average daily alcohol consumption. Participants were then grouped into 4 categories of average daily alcohol intake, using lower cutoffs in women: nondrinkers $(0 \mathrm{~g} / \mathrm{d})$, light $(\leq 5 / 10 \mathrm{~g} / \mathrm{d})$, moderate $(5-20 / 10-40 \mathrm{~g} / \mathrm{d})$, and heavy drinkers ( $\geq 20 / 40 \mathrm{~g} / \mathrm{d}$ ). Third, binge drinking was defined as consuming $\geq 60$ and $100 \mathrm{~g}$ of ethanol in women and men in one episode at least monthly. Baseline information on long-term abstention was not available. Therefore, for all measures, participants reporting no alcohol consumption in the past year were categorized as nondrinkers. Weekly alcohol consumption and alcohol intake in the last 3 months were also available, but were not used for these analyses, because they correlated strongly with the GFQ.

Finally, using average daily alcohol intake at baseline and reexamination, participants were categorized into the following: stable nondrinkers (consistently abstained), ex-drinkers (abstained at follow-up but not baseline), stable drinkers (stable consumption), reduced drinking (higher consumption at baseline), increased drinking (higher consumption at follow-up), and those who started drinking (abstained at baseline but not follow-up).

Problem drinking (assessed 2002-2005 and 2006-2008). Problem drinking was assessed by the CAGE (cutting down, annoyance by criticism, guilty feeling, and eye-openers) questionnaire $^{17}$ on both survey occasions. Positive responses to 2 or more items indicate potential problem drinking.

Covariates (assessed 2002-2005 and 2006-2008). Covariates included study center, age, education (primary or less, vocational, secondary, and university), household asset index (microwave, dishwasher, washing machine, color TV, car, freezer, satellite TV, VCR, camcorder, mobile phone and telephone; range $0-11$ for the number of items owned), smoking status (never, current, former), leisure-time physical activity (none vs some), high depressive symptoms (yes vs no) measured by the CESD-10 (Center for Epidemiologic Studies Depression Scale), and self-reported chronic conditions (CVD, diabetes, and hypertension; all yes vs no). Because some of the health conditions could either mediate or confound the association between alcohol and cognition, we show analyses unadjusted and adjusted for chronic conditions.

Statistical analysis. Cognitive test results obtained at reexamination, the main outcome of these analyses, were converted to $z$ scores (mean $=0 ; S D=1$ ) using whole sample means and SDs to allow comparison between tests. Cross-sectional and prospective associations between alcohol and cognitive 
Table 1 Descriptive characteristics of the study sample

\begin{tabular}{|c|c|c|c|c|}
\hline & \multicolumn{2}{|c|}{ Baseline (2002-2005) } & \multicolumn{2}{|c|}{ Reexamination (2006-2008) } \\
\hline & $\operatorname{Men}(n=6,608)$ & Women $(n=7,967)$ & $\operatorname{Men}(n=6,608)$ & Women $(n=7,967)$ \\
\hline Age, $y$, mean (SD) & $57.8(7.0)$ & $57.3(7.0)$ & $61.3(7.0)$ & $60.8(6.9)$ \\
\hline \multicolumn{5}{|l|}{ Alcohol use, $n(\%)$} \\
\hline Nondrinker & $857(13.0)$ & $1,919(24.1)$ & 749 (11.3) & $1,651(20.7)$ \\
\hline Light & 3,299 (49.9) & $4,736(59.4)$ & 3,518 (53.2) & 4,964 (62.3) \\
\hline Moderate & 2,088 (31.6) & $1,027(12.9)$ & $1,815(27.5)$ & 1,099 (13.8) \\
\hline Heavy & $364(5.5)$ & 285 (3.6) & $526(8.0)$ & 253 (3.2) \\
\hline \multicolumn{5}{|l|}{ Drinking frequency, $\mathrm{n}(\%)$} \\
\hline Never & $857(13.0)$ & $1,919(24.1)$ & 749 (11.3) & 1,651 (20.7) \\
\hline$<1 \mathrm{mo}$ & 919 (13.9) & $2,690(33.8)$ & 939 (14.2) & 3,129 (39.3) \\
\hline $1-3 \mathrm{mo}$ & 1,524 (23.1) & $2,095(26.3)$ & $1,670(25.3)$ & $1,920(24.1)$ \\
\hline 1-4 wk & 2,166 (32.8) & $1,022(12.8)$ & 2,225 (33.7) & 1,053 (13.2) \\
\hline $5+w k$ & $1,142(17.3)$ & 241 (3.0) & $1,025(15.5)$ & $214(2.7)$ \\
\hline \multicolumn{5}{|l|}{ Binge drinking, $n$ (\%) } \\
\hline Nondrinker & 857 (13.0) & $1,919(24.1)$ & 749 (11.3) & $1,651(20.7)$ \\
\hline Nonbinger & $4,540(68.7)$ & $5,590(70.2)$ & $4,471(67.7)$ & $5,799(72.8)$ \\
\hline Binge drinker & $1,211(18.3)$ & $458(5.7)$ & $1,388(21.0)$ & $517(6.5)$ \\
\hline \multicolumn{5}{|l|}{ CAGE, n (\%) } \\
\hline Nondrinker & $857(13.0)$ & $1,919(24.1)$ & 749 (11.3) & $1,651(20.7)$ \\
\hline CAGE $<2$ & $4,963(75.1)$ & $5,936(74.5)$ & $5,022(76.0)$ & $6,213(78.0)$ \\
\hline CAGE 2+ & 788 (11.9) & $112(1.4)$ & 837 (12.7) & $103(1.3)$ \\
\hline Household asset score, mean (SD) & $6.3(2.1)$ & $5.7(2.1)$ & $6.7(2.0)$ & $6.2(2.0)$ \\
\hline \multicolumn{5}{|l|}{ Educational level, n (\%) } \\
\hline Primary or less & $482(7.3)$ & $858(10.8)$ & & \\
\hline Vocational & $1,871(28.3)$ & $1,995(25.0)$ & & \\
\hline Secondary & 2,220 (33.6) & 3,202 (40.2) & & \\
\hline University & 2,035 (30.8) & $1,912(24.0)$ & & \\
\hline \multicolumn{5}{|l|}{ Smoking status, $\mathrm{n}(\%)$} \\
\hline Never smoked & $2,045(30.9)$ & $5,363(67.3)$ & $2,048(31.0)$ & $5,497(69.0)$ \\
\hline Former smoker & $2,164(32.7)$ & $1,167(14.6)$ & 2,354 (35.6) & $1,220(15.3)$ \\
\hline Current smoker & 2,399 (36.3) & $1,437(18.0)$ & 2,206 (33.4) & $1,250(15.7)$ \\
\hline CVD, n (\%) & $1,317(19.9)$ & $1,295(16.3)$ & $1,488(22.5)$ & $1,481(18.6)$ \\
\hline Hypertension, n (\%) & $3,273(49.5)$ & $4,432(55.6)$ & $3,694(55.9)$ & $4,888(61.4)$ \\
\hline Diabetes, n (\%) & 608 (9.2) & $608(7.6)$ & 671 (10.2) & 699 (8.8) \\
\hline \multicolumn{5}{|l|}{ Leisure-time physical activity, $\mathrm{n}(\%)$} \\
\hline None & $2,808(42.5)$ & $3,609(45.3)$ & 2,236 (33.8) & 3,099 (38.9) \\
\hline Some & $3,800(57.5)$ & $4,358(54.7)$ & $4,372(66.2)$ & $4,868(61.1)$ \\
\hline High depressive symptoms, n (\%) & $692(10.5)$ & $1,681(21.1)$ & $843(12.8)$ & $1,976(24.8)$ \\
\hline Immediate recall, mean (SD) & & & $21.5(4.2)$ & $23.0(3.8)$ \\
\hline Delayed recall, mean (SD) & & & $7.3(1.9)$ & $8.0(1.8)$ \\
\hline Verbal fluency, mean (SD) & & & $22.3(7.2)$ & $22.2(7.0)$ \\
\hline Letter cancellation, mean (SD) & & & $17.2(4.8)$ & $18.5(5.0)$ \\
\hline
\end{tabular}

Abbreviations: CAGE = cutting down, annoyance by criticism, guilty feeling, and eye-openers; CVD = cardiovascular disease.

Figures are means (SDs) for continuous variables and counts (\%) for categorical variables. For categories of average daily alcohol intake (lower cutoffs are for women): nondrinker $0 \mathrm{~g}$, light $\leq 5 / 10 \mathrm{~g}$, moderate 5-20/10-40 g, and heavy $\geq 20 / 40 \mathrm{~g}$. 
Table 2 Regression results for alcohol consumption and standardized cognitive scores in men $(n=6,608)$

Immediate recall

Coef. $\quad 95 \% \mathrm{Cl}$

Prospective

Average alcohol use

$$
0 \mathrm{~g} / \mathrm{d}
$$

$<10 \mathrm{~g} / \mathrm{d}$ (ref.)

$10-40 \mathrm{~g} / \mathrm{d}$

$>40 \mathrm{~g} / \mathrm{d}$

Drinking frequency

\section{Never}

$<1$ mo (ref.)

1-3 mo

1-4 wk

$5+w k$

Binge drinking

\section{Nondrinker}

Nonbinger (ref.)

Binge drinker

CAGE

Nondrinker

CAGE <2 (ref.)

CAGE 2+

Cross-sectional

Average alcohol use

$$
0 \mathrm{~g} / \mathrm{d}
$$

$<10$ g/d (ref.)

$10-40 \mathrm{~g} / \mathrm{d}$

$>40 \mathrm{~g} / \mathrm{d}$

Drinking frequency

\section{Never}

$<1$ mo (ref.)

1-3 mo

1-4 wk

$5+w k$

Binge drinking

\section{Nondrinker}

Nonbinger (ref.)

Binge drinker

CAGE

Nondrinker

CAGE <2 (ref.)

CAGE 2+

Follow-up

$\begin{array}{rrr}-0.06 & -0.13,0.01 & -0.08^{a} \\ 0.00 & 0.00,0.00 & 0.00 \\ -0.02 & -0.07,0.03 & -0.03 \\ 0.01 & -0.09,0.11 & -0.01\end{array}$

$\begin{array}{rr}-0.15,-0.01 & -0.05 \\ 0.00,0.00 & 0.00 \\ -0.08,0.02 & 0.01 \\ -0.11,0.09 & -0.08\end{array}$

$-0.12,0.02$

$0.00,0.00$

$-0.04,0.07$

$-0.18,0.02$

$-0.07$

$-0.16,0.01$

$0.00,0.00$

$-0.04$

$-0.11,0.04$

$-0.09,0.05$

0.01

$-0.07,0.09$

$-0.06$

$-0.12,0.01$

0.00

$-0.01$

$0.00,0.00$

$-0.07,0.05$

$-0.06$

$-0.12,0.01$

$0.00,0.00$

$-0.00$

$-0.07,0.07$

$\begin{array}{rr}-0.08 & -0.16,0.01 \\ 0.00 & 0.00,0.00 \\ -0.02 & -0.10,0.05 \\ 0.01 & -0.06,0.08 \\ 0.00 & -0.08,0.08\end{array}$

0.00

$-0.08^{a}$

0.00

$-0.03$

$-0.08^{a}$

0.00

$-0.03$

$-0.15,-0.01$

$0.00,0.00$

$-0.09,0.03$

$-0.15,-0.01$

$0.00,0.00$

$-0.10,0.04$
Verbal fluency

Coef. $\quad 95 \% \mathrm{Cl}$
Letter cancellation

Coef. $\quad 95 \% \mathrm{Cl}$ 
Table 2 Continued

\begin{tabular}{|c|c|c|c|c|c|c|c|c|}
\hline & \multicolumn{2}{|c|}{ Immediate recall } & \multicolumn{2}{|c|}{ Delayed recall } & \multicolumn{2}{|c|}{ Verbal fluency } & \multicolumn{2}{|c|}{ Letter cancellation } \\
\hline & Coef. & $95 \% \mathrm{Cl}$ & Coef. & $95 \% \mathrm{Cl}$ & Coef. & $95 \% \mathrm{Cl}$ & Coef. & $95 \% \mathrm{Cl}$ \\
\hline Stable drinker (ref.) & 0.00 & $0.00,0.00$ & 0.00 & $0.00,0.00$ & 0.00 & $0.00,0.00$ & 0.00 & $0.00,0.00$ \\
\hline Reduced use & -0.02 & $-0.08,0.05$ & -0.00 & $-0.07,0.06$ & $-0.07^{a}$ & $-0.13,-0.01$ & 0.04 & $-0.03,0.10$ \\
\hline Increased use & -0.03 & $-0.09,0.03$ & -0.03 & $-0.10,0.03$ & $-0.07^{a}$ & $-0.14,-0.01$ & 0.00 & $-0.06,0.07$ \\
\hline Started drinking & -0.04 & $-0.13,0.05$ & -0.06 & $-0.16,0.03$ & -0.04 & $-0.13,0.05$ & 0.02 & $-0.07,0.11$ \\
\hline
\end{tabular}

Abbreviations: CAGE = cutting down, annoyance by criticism, guilty feeling, and eye-openers; $\mathrm{Cl}=$ confidence interval; coef. $=$ coefficient; ref. $=$ reference. Models are adjusted for age, center, cognitive measurement occasion, education, household asset score, smoking, physical activity, and depressive symptoms.

${ }^{a} p<0.05$.

${ }^{b}$ Overall $p$ value for alcohol categories by study center interaction significant at $<0.05$.

${ }^{c} p<0.001$.

${ }^{d} p<0.01$.

functions were estimated using multiple linear regression. In cross-sectional analyses, only variables measured at reexamination were used; in prospective analyses, we used cognition from reexamination and alcohol and covariates from baseline. All regression analyses were adjusted for cognitive measurement occasion (first vs repeated).

Analyses were conducted separately by sex because of large differences in alcohol consumption between men and women. To formally test for heterogeneity, alcohol-sex interactions were included in regression models. Heterogeneity of associations between study centers was examined by including interactions between alcohol measures and center, and by conducting analyses stratified by center. For alcohol indices, the lowest level of drinking was used as the reference group. In additional analyses, models for binge drinking were further adjusted for, or stratified by, alcohol volume. Sensitivity analyses were conducted to account for missing covariate data and attrition. Analyses were conducted in Stata 13. ${ }^{18}$

RESULTS Complete data on all cognitive tests were available for 17,022 participants in 2006-2008, 14,575 of whom also had data on alcohol and all covariates and were included in the analytic sample. Table 1 shows baseline and follow-up characteristics of included participants (descriptive statistics by alcohol use categories are shown in table e-1 on the Neurology ${ }^{\circledR}$ Web site at Neurology.org). Proportions of binge drinkers and problem drinkers as well as average alcohol use were higher in men than in women; baseline median daily alcohol intake was 5 . 3 and $0.6 \mathrm{~g}$, respectively.

Baseline and follow-up alcohol measures were strongly associated with each other. Spearman coefficients were 0.62 and 0.58 for quantity and frequency of drinking. Tetrachoric coefficient for binge drinking was 0.60 . For categories of alcohol intake, $58 \%$ of participants were in the same group on both occasions, and $44 \%$ of participants were in the same or adjacent category for drinking frequency.

In regression analyses, heterogeneity between centers was observed in only a few instances and was mostly weak. In fully adjusted models, several alcohol-sex interactions, especially for recall tests, were evident at $p<0.05$. Results of regression analyses adjusted for potential confounders are shown in table 2 (men) and table 3 (women). Age-adjusted regression results are available in the online supplement (table e-2 [men] and table e-3 [women]). In men, the quantity and frequency of drinking were generally not significantly associated with cognitive test scores, either prospectively or cross-sectionally, after adjusting for socioeconomic and lifestyle confounders. The only consistent exception was lower cognitive scores in nondrinkers compared with the reference group in cross-sectional models.

In women, nondrinkers had consistently lower cognitive scores than light drinkers in both prospective and cross-sectional analyses. Better cognitive performance in moderate drinkers observed in ageadjusted models (table e-3) was largely attenuated by subsequent adjustment for socioeconomic and lifestyle factors. In cross-sectional analyses, moderate drinking frequency and intake were associated with better scores, compared with lower levels of drinking. In prospective analyses, only women drinking a few times a month showed better performance on the verbal cognitive tests than those who drank less frequently than that, whereas average intake was not associated with better cognition.

Binge drinking was not significantly associated with cognitive performance in either sex in prospective analyses; cross-sectional associations were also few and inconsistent. CAGE was also not related to cognitive performance, although in women, the number of problem drinkers was relatively small.

Results for drinking pattern, measured by combining data on frequency and quantity per occasion, did not yield any additional insights and are reported in the online supplement separately for men (table e-4) and women (table e-5).

Results for change in alcohol intake during follow-up showed that, especially in men, participants who stopped 
Table 3 Regression results for alcohol consumption and standardized cognitive scores in women $(n=7,967)$

Immediate recall

Coef. $\quad 95 \% \mathrm{Cl}$

Delayed recall

Coef. $\quad 95 \% \mathrm{Cl}$

Verbal fluency
Coef. $\quad 95 \% \mathrm{Cl}$

Letter cancellation

Coef. $\quad 95 \% \mathrm{Cl}$

Prospective

Average alcohol use

$$
0 \mathrm{~g} / \mathrm{d}
$$

$<5 \mathrm{~g} / \mathrm{d}$ (ref.)

5-20 g/d

$>20 \mathrm{~g} / \mathrm{d}$

Drinking frequency

\section{Never}

$<1$ mo (ref.)

1-3 mo

1-4 wk

$5+w k$

Binge drinking

\section{Nondrinker}

Nonbinger (ref.)

Binge drinker

CAGE

Nondrinker

CAGE <2 (ref.)

CAGE 2+

Cross-sectional

\section{Average alcohol use}

\section{$0 \mathrm{~g} / \mathrm{d}$}

$<5 \mathrm{~g} / \mathrm{d}$ (ref.)

5-20 g/d

$>20 \mathrm{~g} / \mathrm{d}$

Drinking frequency

\section{Never}

$<1$ mo (ref.)

1-3 mo

1-4 wk

$5+w k$

\section{Binge drinking}

\section{Nondrinker}

Nonbinger (ref.)

Binge drinker

CAGE

Nondrinker

CAGE <2 (ref.)

CAGE 2+

Follow-up

$-0.14^{a}$
0.00
-0.01
-0.02

$-0.19,-0.09$
$0.00,0.00$
$-0.07,0.05$
$-0.13,0.09$

$-0.11^{a}$

$-0.17,-0.06$

$-0.11^{a}$

0.00

$0.08^{b}$

0.03

0.02

$-0.16,-0.05$

0.00, 0.00

$0.03,0.13$

$-0.04,0.10$

$-0.10,0.14$

$-0.14^{a}$

0.00

$-0.05$

$-0.14^{a}$

0.00

$-0.15$

$-0.19,-0.09$

$0.00,0.00$

$-0.13,0.04$

$-0.19,-0.09$

$0.00,0.00$

$-0.32,0.01$
0.00

0.05

0.03

$0.00,0.00$

$-0.01,0.11$

$-0.08,0.14$

$-0.09^{b}$

0.00

$0.06^{d}$

$0.07^{d}$

0.05

$-0.15,-0.04^{c}$

$0.00,0.00$

0.00, 0.11

$0.00,0.14$

$-0.07,0.17$

$-0.12^{a}$

0.00

0.01

$-0.17,-0.07$

$0.00,0.00$

$-0.08,0.10$

$-0.13^{a}$

0.00

$-0.13$

$-0.18,-0.08$

$0.00,0.00$

$-0.30,0.03$ $-0.12^{a}$

0.00

$-0.02$

0.05

$-0.08^{b}$

0.00

$0.09^{b}$

0.04

0.08

$-0.12^{a}$

0.00

0.00

$-0.12^{a}$

0.00

0.04
$-0.17,-0.07$

$0.00,0.00$

$-0.08,0.04$

$-0.06,0.16$

$-0.14-0.03$

0.00, 0.00

$0.03,0.14$

$-0.03,0.10$

$-0.04,0.20$

$-0.17,-0.07$

$0.00,0.00$

$-0.08,0.09$

$-0.17,-0.07$

$0.00,0.00$

$-0.13,0.21$ $-0.14^{a}$

0.00

$-0.02$

0.00

$-0.13^{a}$

0.00

0.03

0.01

0.03

$-0.14^{a}$

0.00

$-0.07$

$-0.14^{a}$

0.00

$-0.15$
$-0.20,-0.09$

$0.00,0.00$

$-0.08,0.05$

$-0.11,0.12$ 
Table 3 Continued

\begin{tabular}{|c|c|c|c|c|c|c|c|c|}
\hline & \multicolumn{2}{|c|}{ Immediate recall } & \multicolumn{2}{|c|}{ Delayed recall } & \multicolumn{2}{|c|}{ Verbal fluency } & \multicolumn{2}{|c|}{ Letter cancellation } \\
\hline & Coef. & $95 \% \mathrm{Cl}$ & Coef. & $95 \% \mathrm{Cl}$ & Coef. & $95 \% \mathrm{Cl}$ & Coef. & $95 \% \mathrm{Cl}$ \\
\hline Stable drinker (ref.) & 0.00 & $0.00,0.00$ & 0.00 & $0.00,0.00$ & 0.00 & $0.00,0.00$ & 0.00 & $0.00,0.00$ \\
\hline Reduced use & -0.03 & $-0.10,0.05$ & -0.00 & $-0.08,0.07$ & -0.03 & $-0.11,0.04$ & -0.03 & $-0.10,0.05$ \\
\hline Increased use & 0.07 & $-0.01,0.14$ & 0.05 & $-0.02,0.13$ & 0.01 & $-0.07,0.08$ & 0.02 & $-0.05,0.10$ \\
\hline Started drinking & $-0.11^{a}$ & $-0.17,-0.04$ & $-0.10^{b}$ & $-0.17,-0.04$ & $-0.10^{b}$ & $-0.16,-0.03$ & $-0.09^{b}$ & $-0.16,-0.02$ \\
\hline
\end{tabular}

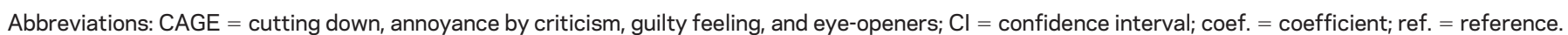

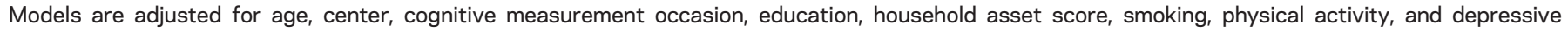
symptoms.

${ }^{a} p<0.001$

${ }^{b} p<0.01$.

${ }^{c}$ Overall $p$ value for alcohol categories by country interaction significant at $<0.05$.

${ }^{d} p<0.05$.

drinking had significantly lower cognitive scores than stable drinkers. There were almost no statistically significant differences between stable drinkers and other drinkers. In women, stable nondrinkers and those who started drinking during follow-up also had lower cognitive scores. In men, lower verbal performance in stable nondrinkers was marginally significant. Results for change in drinking frequency or combining information on frequency and intake during follow-up were similar (table e-6).

Finally, additional adjustment for health conditions resulted in little change in associations with cognition for all alcohol measures (tables e-7 and e-8).

Results of sensitivity analyses using inverse probability weighting to account for longitudinal attrition (table e-9) and multiple imputation to account for missing covariate data (tables e-10 and e-11) are available in the online supplement.

DISCUSSION In this large Eastern European study with relatively high levels of male regular and episodic heavy drinking, we found only modest associations between different measures of alcohol consumption and cognitive performance in older persons. Quantity and frequency of drinking were not associated with cognitive performance in men, whereas in women, moderate drinkers had slightly better cognition than those drinking less, particularly in cross-sectional analyses. The most consistent findings were worse cognitive performance in female nondrinkers and those who stopped drinking during follow-up. Binge and problem drinking were not consistently associated with cognitive function.

Our study has limitations. First, the analytic sample was restricted to participants with cognitive measures at follow-up. Attrition was higher in participants with lower cognitive scores, nondrinkers, and frequent drinkers at baseline, and could possibly bias the results of our study. However, sensitivity analysis accounting for missing cognition data by inverse probability weighting yielded similar results (table e-6).

Second, self-reported alcohol measures typically underestimate actual consumption. ${ }^{19}$ In this study, systematic underreporting among men seems unlikely but, because of sex differences in social acceptability of drinking, underreporting may be greater in women. ${ }^{20}$ Among available alcohol measures, the GFQ appeared to be least prone to underreporting. In addition, correlations between different alcohol measures were generally high, and serum GGT $(\gamma$-glutamyltransferase) values showed expected associations with alcohol, although the correlations were weaker in women.

Third, alcohol measures used may not fully reflect lifelong consumption. Particularly, participants may have stopped or reduced drinking before the baseline, and those who stopped drinking during follow-up had worse cognition than stable drinkers. However, unstable drinkers generally did not differ from stable drinkers, and the few significant differences may be attributable to chance. While this would suggest that results for drinkers were not affected by inclusion of those who had reduced their consumption, it is also possible that regression to the mean affected our estimates in relation to change in alcohol consumption, leading to regression dilution bias. However, this does not affect our prospective and cross-sectional results.

Fourth, response rates were relatively low and responders were healthier than nonresponders. ${ }^{15}$ While this is also true of most contemporary studies, it suggests that heavier drinkers and individuals with cognitive impairment may have been less likely to participate. Low response and differences in characteristics between responders and nonresponders do not inevitably bias estimates of associations. ${ }^{21}$ However, if in our study heavy drinkers had lower participation rates and higher likelihood of cognitive 
impairment, it is possible that associations between heavy drinking and cognitive function were underestimated.

Finally, the study did not examine nationally representative samples and extrapolation beyond urban settings requires caution.

A particular strength of this study is the use of Eastern European population samples with relatively high alcohol consumption and rates of male binge drinking in the former Soviet Union, ${ }^{22}$ and a higher proportion of CVD and premature mortality estimated to be attributable to alcohol than elsewhere in Europe. ${ }^{14}$ Indeed, our study had a high prevalence of male binge and problem drinking. Second, the GFQ is well suited for identifying heavy and binge drinkers. ${ }^{16}$ Third, the large numbers in the pooled sample provide adequate statistical power in men, although the numbers of heavy drinkers were small in women. Finally, unlike most previous studies, our emphasis was on the cognitive effects of regular and episodic heavy drinking, not just light to moderate drinking.

We did not find convincing evidence of worse cognitive performance in heavy drinkers. More specific tests of executive function could show greater sensitivity to the effects of alcohol, as previously reported for phonemic fluency. ${ }^{3}$ A study in British civil servants reported faster cognitive decline in middle-aged men drinking $\geq 36 \mathrm{~g} / \mathrm{d}$, compared with $<20 \mathrm{~g} / \mathrm{d}$, but no effect in women. ${ }^{8}$ It is possible that regular and/or episodic heavy alcohol use is associated with faster cognitive decline in Eastern European populations, but we do not yet have a long enough follow-up to examine trajectories of cognitive decline.

In our study, binge drinking was not consistently associated with cognitive performance. We were therefore unable to replicate findings from one study of Finish twins, in which binge drinking was independently associated with cognitive impairment ${ }^{10}$ and dementia. ${ }^{11}$

The main hypothesized mechanism for the association between alcohol and cognition involves effects on the cardio- and cerebrovascular systems. ${ }^{13}$ Heavy drinking and abstention have been linked to higher cardiovascular risk than light to moderate drinking, and binge drinking may independently increase cardiovascular risk. ${ }^{12}$ In a prospective study in Novosibirsk, regular but not episodic heavy drinking increased risk of cardiovascular death. ${ }^{23}$ Binge drinking was also not consistently associated with high blood pressure ${ }^{24}$ or all-cause and cardiovascular mortality in this cohort (unpublished findings). Thus, the absence of association between binge drinking and cognitive performance could plausibly reflect the apparent lack of adverse effects of binge drinking on CVD in this cohort.
Most observational studies report moderate drinking to be beneficial for cognitive function in mid- to later life ${ }^{2}$ with anti-inflammatory effects postulated among the underlying mechanisms. ${ }^{25}$ Consistent with this, we observed significantly better cognitive scores in moderate drinkers than low-level drinkers in women. However, compared with cross-sectional results, the prospective associations were modest and inconsistent. This discrepancy suggests that reverse causality, selection, or confounding by social or lifestyle factors may partly account for the positive correlation between moderate drinking and cognitive performance, as previously suggested. ${ }^{26,27} \mathrm{~A}$ causal association was also not supported by negative findings of mendelian randomization studies in Chinese men $^{28}$ and Europeans. ${ }^{29}$ However, a recent study in a Scottish birth cohort suggested that the effects of alcohol on late-life cognition may be modified by genetic ability to metabolize alcohol. ${ }^{30}$

Similarly, lower cognitive scores in participants who stopped drinking during follow-up may partly reflect confounding by poor health. In fact, at reexamination, more than half of these participants reported stopping drinking because of poor health. However, adjustment for self-reported chronic conditions had little effect on the associations.

Our study has implications. Better cognitive performance was only inconsistently associated with moderate drinking, with some of the associations possibly explained by selection bias or confounding. This cautions against specific recommendations about beneficial drinking levels for cognitive function. Poorer cognition in current nondrinkers and recent quitters suggests greater attention should be given to the health status of this group. Although adverse effects of binge drinking on cognition were not detected over a short follow-up period or cross-sectionally, our findings do not preclude the possibility of faster cognitive decline in the long term among binge drinkers.

\section{AUTHOR CONTRIBUTIONS}

M.B. and P.H. conceptualized the study. P.H. performed statistical analysis and wrote first and successive drafts of manuscript. M.B., M.R., R.K., A. Pajak, S.M., S.S., H.P., A. Peasey, M.M., and A.S.-M. interpreted the results and commented on drafts.

\section{STUDY FUNDING}

This work was supported by the Wellcome Trust (grants 064947 and 081081); the US National Institute on Aging (grant R01 AG23522); and MacArthur Foundation Research Network (grant 712058).

\section{DISCLOSURE}

P. Horvat reports no disclosures relevant to the manuscript. M. Richards receives research support from the UK Medical Research Council and the British Heart Foundation. R. Kubinova, A. Pajak, S. Malyutina, S. Shishkin, H. Pikhart, A. Peasey, and M. Marmot report no disclosures relevant to the manuscript. A. Singh-Manoux receives research support from the US NIH (R01AG013196; R01AG034454). M. Bobak reports no disclosures relevant to the manuscript. Go to Neurology.org for full disclosures. 
15. Peasey A, Bobak M, Kubinova R, et al. Determinants of cardiovascular disease and other non-communicable dis-

\section{REFERENCES}

1. Amieva H, Jacqmin-Gadda H, Orgogozo JM, et al. The 9 year cognitive decline before dementia of the Alzheimer type: a prospective population-based study. Brain 2005; 128:1093-1101.

2. Neafsey EJ, Collins MA. Moderate alcohol consumption and cognitive risk. Neuropsychiatr Dis Treat 2011;7:465-484.

3. Gross AL, Rebok GW, Ford DE, et al. Alcohol consumption and domain-specific cognitive function in older adults: longitudinal data from the Johns Hopkins Precursors Study. J Gerontol B Psychol Sci Soc Sci 2011;66:39-47.

4. Bond GE, Burr R, McCurry SM, Graves AB, Larson EB. Alcohol, aging, and cognitive performance in a cohort of Japanese Americans aged 65 and older: the Kame Project. Int Psychogeriatr 2001;13:207-223.

5. Britton A, Singh-Manoux A, Marmot M. Alcohol consumption and cognitive function in the Whitehall II Study. Am J Epidemiol 2004;160:240-247.

6. Sabia S, Gueguen A, Berr C, et al. High alcohol consumption in middle-aged adults is associated with poorer cognitive performance only in the low socio-economic group: results from the GAZEL cohort study. Addiction 2011; 106:93-101.

7. Richards M, Hardy R, Wadsworth ME. Alcohol consumption and midlife cognitive change in the British 1946 birth cohort study. Alcohol Alcohol 2005;40:112-117.

8. Sabia S, Elbaz A, Britton A, et al. Alcohol consumption and cognitive decline in early old age. Neurology 2014;82: 332-339.

9. Anstey KJ, Mack HA, Cherbuin N. Alcohol consumption as a risk factor for dementia and cognitive decline: metaanalysis of prospective studies. Am J Geriatr Psychiatry 2009; 17:542-555.

10. Virtaa JJ, Jarvenpaa T, Heikkila K, et al. Midlife alcohol consumption and later risk of cognitive impairment: a twin follow-up study. J Alzheimers Dis 2010;22:939-948.

11. Jarvenpaa T, Rinne JO, Koskenvuo M, Raiha I, Kaprio J. Binge drinking in midlife and dementia risk. Epidemiology 2005;16:766-771.

12. Rehm J, Sempos CT, Trevisan M. Alcohol and cardiovascular disease: more than one paradox to consider. Average volume of alcohol consumption, patterns of drinking and risk of coronary heart disease- a review. J Cardiovasc Risk 2003;10:15-20.

13. Spiro A, Brady CB. Integrating health into cognitive aging: toward a preventive cognitive neuroscience of aging. J Gerontol B Psychol Sci Soc Sci 2011;66:17-25.

14. Rehm J, Sulkowska U, Manczuk M, et al. Alcohol accounts for a high proportion of premature mortality in Central and Eastern Europe. Int J Epidemiol 2007;36: 458-467. eases in Central and Eastern Europe: rationale and design of the HAPIEE Study. BMC Public Health 2006;6:255.

16. Rehm J. Measuring quantity, frequency, and volume of drinking. Alcohol Clin Exp Res 1998;22:4S-14S.

17. Ewing JA. Detecting alcoholism: the CAGE questionnaire. JAMA 1984;252:1905-1907.

18. Stata Statistical Software: Release 13 [Computer Program]. College Station, TX: StataCorp LP; 2013.

19. Stockwell T, Donath S, Cooper-Stanbury M, Chikritzhs T, Catalano P, Mateo C. Under-reporting of alcohol consumption in household surveys: a comparison of quantity-frequency, graduated-frequency and recent recall. Addiction 2004;99:1024-1033.

20. Bobrova N, West R, Malyutina D, Malyutina S, Bobak M. Gender differences in drinking practices in middle aged and older Russians. Alcohol Alcohol 2010;45: 573-580.

21. Stang A. Nonresponse research: an underdeveloped field in epidemiology. Eur J Epidemiol 2003;18:929-932.

22. Popova S, Rehm J, Patra J, Zatonski W. Comparing alcohol consumption in Central and Eastern Europe to other European countries. Alcohol Alcohol 2007;42:465-473.

23. Malyutina S, Bobak M, Kurilovitch S, et al. Relation between heavy and binge drinking and all-cause and cardiovascular mortality in Novosibirsk, Russia: a prospective cohort study. Lancet 2002;360:1448-1454.

24. Pajak A, Szafraniec K, Kubinova R, et al. Binge drinking and blood pressure: cross-sectional results of the HAPIEE Study. PLoS One 2013;8:e65856.

25. Collins MA, Neafsey EJ, Mukamal KJ, et al. Alcohol in moderation, cardioprotection, and neuroprotection: epidemiological considerations and mechanistic studies. Alcohol Clin Exp Res 2009;33:206-219.

26. Corley J, Jia XL, Brett CE, et al. Alcohol intake and cognitive abilities in old age: the Lothian Birth Cohort 1936 Study. Neuropsychology 2011;25:166-175.

27. Cooper C, Bebbington P, Meltzer H, et al. Alcohol in moderation, premorbid intelligence and cognition in older adults: results from the Psychiatric Morbidity Survey. J Neurol Neurosurg Psychiatry 2009;80:1236-1239.

28. Yeung SLA, Jiang CQ, Cheng KK, et al. Evaluation of moderate alcohol use and cognitive function among men using a mendelian randomization design in the Guangzhou Biobank Cohort Study. Am J Epidemiol 2012;175: 1021-1028.

29. Kumari M, Holmes MV, Dale CE, et al. Alcohol consumption and cognitive performance: a mendelian randomization study. Addiction 2014;109:1462-1471.

30. Ritchie SJ, Bates TC, Corley J, et al. Alcohol consumption and lifetime change in cognitive ability: a gene $\times$ environment interaction study. Age 2014;36:9638. 


\section{Neurology}

\section{Alcohol consumption, drinking patterns, and cognitive function in older Eastern European adults}

Pia Horvat, Marcus Richards, Ruzena Kubinova, et al. Neurology 2015;84;287-295 Published Online before print December 12, 2014

DOI 10.1212/WNL.0000000000001164

This information is current as of December 12, 2014

\begin{tabular}{|c|c|}
\hline $\begin{array}{l}\text { Updated Information \& } \\
\text { Services }\end{array}$ & $\begin{array}{l}\text { including high resolution figures, can be found at: } \\
\text { http://n.neurology.org/content/84/3/287.full }\end{array}$ \\
\hline Supplementary Material & $\begin{array}{l}\text { Supplementary material can be found at: } \\
\text { http://n.neurology.org/content/suppl/2014/12/12/WNL.0000000000001 } \\
\text { 164.DC1 }\end{array}$ \\
\hline References & $\begin{array}{l}\text { This article cites } 29 \text { articles, } 2 \text { of which you can access for free at: } \\
\text { http://n.neurology.org/content/84/3/287.full\#ref-list- } 1\end{array}$ \\
\hline Subspecialty Collections & $\begin{array}{l}\text { This article, along with others on similar topics, appears in the } \\
\text { following collection(s): } \\
\text { All epidemiology } \\
\text { http://n.neurology.org/cgi/collection/all_epidemiology } \\
\text { Cognitive aging } \\
\text { http://n.neurology.org/cgi/collection/cognitive_aging } \\
\text { Cohort studies } \\
\text { http://n.neurology.org/cgi/collection/cohort_studies } \\
\text { Risk factors in epidemiology } \\
\text { http://n.neurology.org/cgi/collection/risk_factors_in_epidemiology }\end{array}$ \\
\hline Permissions \& Licensing & $\begin{array}{l}\text { Information about reproducing this article in parts (figures,tables) or in } \\
\text { its entirety can be found online at: } \\
\text { http://www.neurology.org/about/about_the_journal\#permissions }\end{array}$ \\
\hline Reprints & $\begin{array}{l}\text { Information about ordering reprints can be found online: } \\
\text { http://n.neurology.org/subscribers/advertise }\end{array}$ \\
\hline
\end{tabular}

Neurology ${ }^{\circledR}$ is the official journal of the American Academy of Neurology. Published continuously since 1951, it is now a weekly with 48 issues per year. Copyright () 2014 American Academy of Neurology. All rights reserved. Print ISSN: 0028-3878. Online ISSN: 1526-632X.

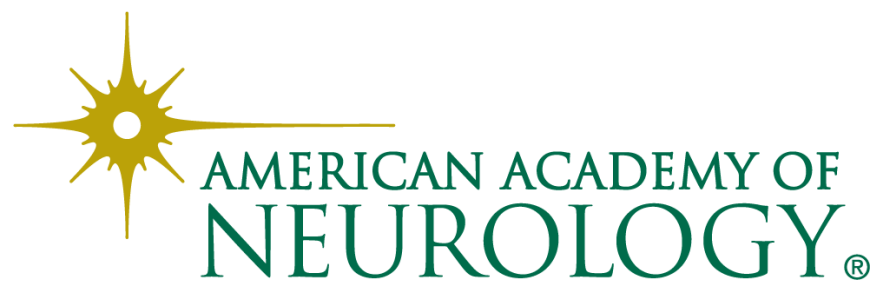

